\title{
ENTREVISTA CON: \\ SANDRA BUECHLER (III) - La persona que es el clínico ${ }^{1}$
}

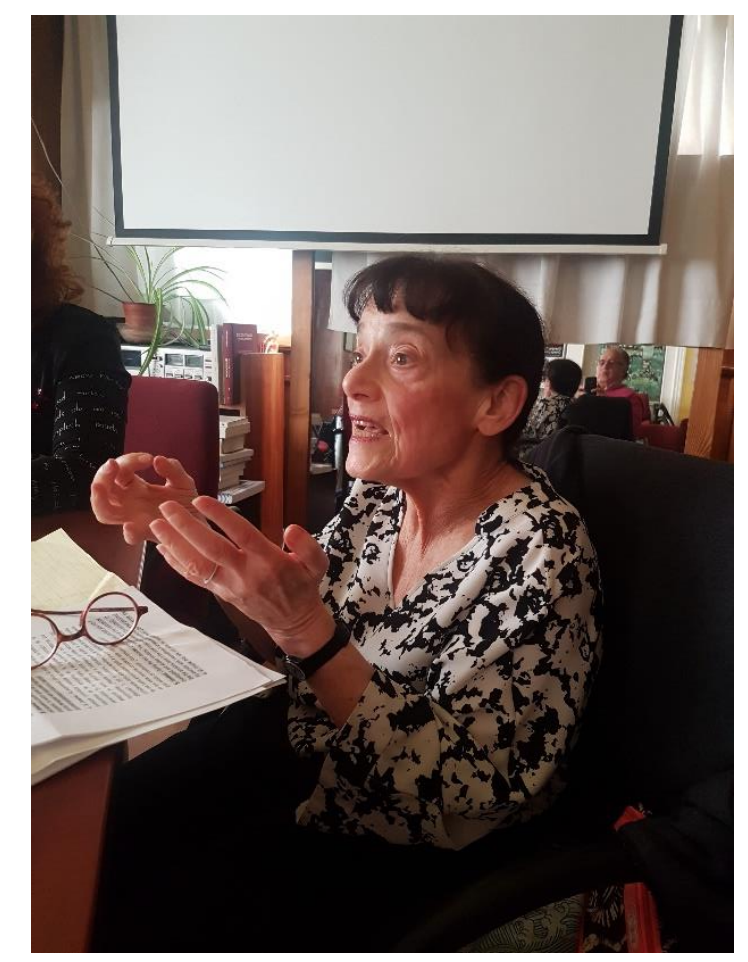

Realizada por Silvia Jiménez Torres, con la participación de Alejandro Ávila, Mario Nervi, Sandra Toribio y Lucía Martínez Domínguez ${ }^{2}$

Silvia Jiménez - Buenas tardes doctora Buechler. Bienvenida nuevamente a Madrid y al Instituto de Psicoterapia Relacional. Estamos encantados de tenerla de vuelta en España, concedernos esta entrevista y querer compartir su tiempo con nosotros. Para no redundar en temas que $\mathrm{Vd}$. ya ha abordado en anteriores entrevistas, en esta ocasión hemos seleccionado algunas preguntas relativas a "La experiencia de vida y el ejercicio profesional de más de cuatro décadas de una psicoanalista", título de esta entrevista. Alejandro tiene una pregunta.

Alejandro Ávila - Vd. ha subrayado la importancia de que los maestros vivan "en voz alta" sus experiencias, para que podamos usarlas en la nuestra propia. Una de las facetas que no ha quedado recogida hasta ahora en sus obras o trabajos son las conexiones entre su propia vida

\footnotetext{
1 Jiménez, S., Ávila, A., Nervi, M., Toribio, S. y Martínez, L. (2020). Entrevista con Sandra Buechler (III) - La persona que es el clínico. Clínica e Investigación Relacional, 14 (1): 255-261. [ISSN 1988-2939] [Recuperado de www.ceir.info] DOI: 10.21110/19882939.2020.140118

2 Traducción de Lucía Martínez Domínguez. Dos entrevistas anteriores realizadas a esta autora fueron publicadas por CeIR en 2015 y 2019.
} 
personal y sus retos en la clínica, solo algunos destellos, muy ricos, pero que no nos permiten conocer su propia vida. Respetamos su privacidad, y no tiene que responder a esto, pero creemos que podría ser útil entender cómo llega vd. a la clínica con su compromiso apasionado y ético, a partir de su vida en su familia de origen, o en sus principales vínculos posteriores. De igual modo, nos gustaría que nos pudiera hablar de lo que ha representado para vd. sus condiciones físicas naturales, y como ha luchado para trascenderlas.

Sandra Buechler: Creo que gran parte de mi experiencia personal me llevó a abrazar algunas de las enseñanzas de H.S. Sullivan. Como él, me sentía muy sola cuando crecía. Inmediatamente entendí su énfasis en la necesidad de conexión, como una especie de requisito absoluto de vida o muerte. Que es tan necesario para la vida humana como el aire, el agua, la comida.

Mis primeros trabajos fueron en hospitales mentales terribles, aterradores y enormes. Empecé a trabajar en estos hospitales a mis 20 años. Rápidamente subí la escalera, y fui un líder del equipo a una edad muy temprana, a cargo de un barrio. Los escritos de Sullivan y Frieda Fromm-Reichmann me ayudaron a sentir que estos pacientes que se comportaban tan extrañamente todavía podían ser entendidos, eran humanos, "más simplemente humanos que otra cosa". Me ayudaron a tener esperanza para mi trabajo. Me sentí muy abrumada. Los métodos de Sullivan de dividir el tratamiento en fases, obtener una historia muy cuidadosa, etc. fueron muy tranquilizadores para mí. Me sentí capaz de hacer el tratamiento de esta manera. Me di cuenta de que podía soportar estar en estos ajustes muy deprimentes si sentía que podía hacer algunas conexiones con los pacientes.

Más tarde, mi primer analista tuvo una influencia muy, muy grande en mi vida y trabajo. Era una mujer con los pies en la tierra, directa, muy empática. Ella me entendió profundamente, muy rápidamente. De ella desarrollé gran parte de mi forma de trabajar y pasión. Ella, realmente, cambió mi vida. Con ella vi en mi propia experiencia cómo el tratamiento puede marcar una gran diferencia en cómo se siente la vida.

Mi analista había tenido poliomielitis al principio de su vida. La dejó gravemente discapacitada. Pero era una luchadora fuerte. A pesar de las probabilidades en su contra, ella luchó por tener una vida plena. De ella aprendí mucho sobre el coraje, sobre lo que es ser realmente entendido, y sobre la belleza de la poesía. Ya me encantaba la poesía, pero ella trajo más de ella a mi vida. Creo que su espíritu, más que el de nadie, es lo que ha dado forma a mis actitudes sobre el trabajo clínico.

Mi segunda analista (analista didacta en el White) también tuvo una influencia muy grande. De ella tomé la dedicación absoluta al trabajo. Estaba totalmente concentrada en nuestro intercambio. También tenía una integridad absoluta. Su lealtad a la verdad fue realmente 
notable. Era una clínica apasionada, trabajaba muy duro, creía en lo que estaba haciendo con todo su corazón. Su énfasis en explorar las defensas, y cómo limitan la vida, tuvo un gran impacto en mí tanto personalmente como en mi propio trabajo clínico.

En cuanto a los problemas físicos que he tenido, la mayor parte de mi carrera estaba básicamente bien, excepto por dos hechos. Es sólo en los últimos años que los problemas físicos me han obstaculizado regularmente.

Las dos excepciones fueron cuando tuve neumonía grave hace muchos años y cuando me diagnosticaron cáncer en 2004. En ambas ocasiones, luché muy duro para recuperar mi camino. En 2004, cuando tuve que pasar por la radiación, le pedí a Don Stern que me diera una tarea, lo cual hizo, y escribí una reseña de libros para Contemporary Psychoanalysis en la sala de espera de la clínica de radiación. Fue una época en la que vi las fortalezas y limitaciones de mi forma de afrontar. Creo que cada uno tiene que luchar con las limitaciones físicas a su manera.

Recientemente, me dijeron que me estaba dañando la espalda sentándome en una silla la mayor parte del día y trabajando. Tuve más y más dolor de espalda severo. Esta es una de las razones por las que me retiré en mayo de 2019. Mientras, hasta ese momento, siempre había luchado para seguir trabajando, en este punto, sentí que me estaba lastimando, y era hora de parar. No fue en absoluto una decisión fácil. Y había otros factores. Volveremos a tratar este tema.

Silvia Jiménez: A propósito de la pregunta de Alejandro, hay una cuestión que me gustaría preguntarle. En mi caso, padezco una enfermedad que me produce pérdida auditiva. ¿Qué considera usted al respecto de comunicarle o no esto al paciente? ¿En qué manera puede influirle?

Sandra Buechler: Antes de tener cáncer, aconsejé a otra analista que me preguntó sobre si le decía a sus pacientes sobre su propio cáncer, que debía tener cuidado, ser conservadora, contar tan poco como se sintiera cómoda ella, porque cualquier cosa que le digas está ahí para siempre, y un año podrías arrepentirte de haber contado. Pero cuando desarrollé cáncer me di cuenta de que, en primer lugar, tenía que decir algo porque perdí sesiones de los tratamientos. Segundo, nunca miento. Eso es un absoluto. $Y$ no digo algo muy vago, como "tengo una cita personal". Como pido a los pacientes que sean directos conmigo, trato de ser tan directa con ellos. Podría decir "Prefiero no entrar en eso" Eso es diferente.

Pero también, a nivel práctico, sólo diciendo la verdad, que tenía cáncer, tenía sentido. No podía decirles a unos más y a otros menos, porque no sabría qué había dicho a quién. Acabe expresa. Este material es para uso científico y profesional exclusivamente y puede contener información clínica sensible. Los editores no se responsabilizan de los contenidos de los autores. Dirigir las consultas sobre derechos y autorizaciones a ceir@psicoterapiarelacional.es 
por decirles a todos una versión breve. Y aprendí que una pregunta puede ser muy diferente, en lo abstracto, de lo que es en la experiencia real!.

Yo también tengo pérdida auditiva. La cual he comunicado a la gente cuando surgió. Creo en decirle a los pacientes cualquier cosa que pueda tener un impacto importante en la sesión. Los principios para mí son primero, honestidad, y segundo, no quiero confundir al paciente acerca de si están percibiendo correctamente. Si creen que no los estoy escuchando, no quiero que se culpen a sí mismos, se confundan acerca de lo que están experimentando, o sientan que no son libres de decir "No creo que me hayas oído".

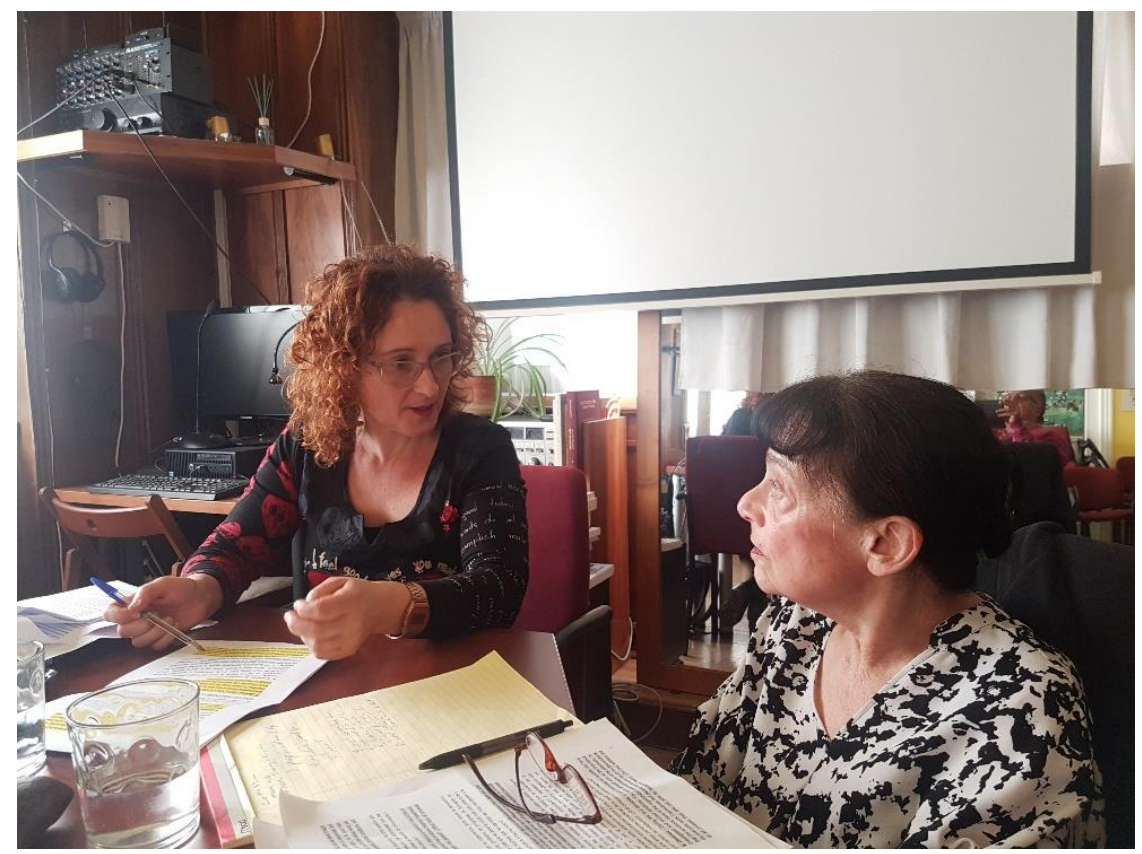

Silvia Jiménez Torres entrevista a Sandra Buechler en Ágora Relacional, Madrid.

Silvia Jiménez: Una persona con su experiencia, tanto desde la perspectiva clínica como académica, como profesora en programas de formación en psicoterapia; ¿En qué áreas cree que se debería incidir más para dotar a los y las jóvenes terapeutas de recursos que les permitan ser más resistentes a la contratransferencia traumática o trauma vicario?

Sandra Buechler: En primer lugar, creo en el punto de vista de que la contratransferencia es principalmente información sobre lo que está pasando en la habitación. Traté de comunicar eso en mi enseñanza. Es información, sobre ambos participantes, que no debe ser utilizada como auto-condena del clínico. Si el clínico no se avergüenza de su contratransferencia, o se siente demasiado culpable por ello, probablemente se ocuparán mejor de ello.

Un segundo aspecto, en mi idea del "coro interno", es que esperaba que el joven clínico interiorizara a supervisores útiles y empáticos durante su formación y más allá. 
Si, como supervisor, piensas en voz alta, de una manera abierta, oirán cuando te abrumas por la situación con el paciente $y$, con suerte, te sientas más capaz de compartir lo que están pasando. Soy una gran fan de la supervisión continua entre pares. Ayudé a crear un grupo de compañeros en 1980 que todavía está activo.

$Y$ creo que es muy importante escribir sobre las expectativas muy altas que nos ponemos como analistas, y que podrían ser poco realistas o causar tensiones y traumas vicarios. Mi libro Still Practicing (Aún ejerciendo) trata en gran medida sobre este tema.

Silvia Jiménez: Su experiencia clínica, de más de cuatro décadas, le ha hecho investigar, profundizar y estudiar las emociones. Usted nos ha transmitido la importancia de las emociones en la organización y expresión de la personalidad. En mi trabajo diario, a veces me encuentro con personas que aseguran no sentir emoción (aunque puede que lo que no sepan sea reconocerlas). ¿Aborda usted el tratamiento con estas personas de manera diferente?. Y, por el contrario, actualmente acuden a consulta personas consideradas como altamente sensibles. Una de sus características esenciales es una fuerte emocionalidad ligada a una gran capacidad empática. La cuestión es que estas personas consideran este rasgo como inmutable y presentan un gran estado de indefensión. ¿Qué considera usted al respecto?

Sandra Buechler: Este es un tema donde mi formación, orientada a notar cambios no verbales muy pequeños, podría ser útil. Fui formada para registrar cualquier ligero cambio en la cara o el cuerpo del paciente como posiblemente significativo. Mis supervisores me ayudaron a notar, por ejemplo, que el paciente está cambiando su cuerpo, y se preguntan si el paciente está ansioso, y preguntan, "¿Qué sientes ahora?" Tienes que coger una emoción, en lo que yo llamo la etapa de "brote", o las primeras etapas. No creo que sea útil, por lo general, preguntar sobre lo que el paciente sintió ayer, o esta semana, si el paciente no está en contacto con los sentimientos. Tienes que atraparlos como suceden en la sesión.

Las personas muy sensibles a menudo están tratando de sentirse menos intensamente y no lo consiguen. Este es, para mí, el enfoque equivocado. Las emociones no son como un termostato que puedes subir o bajar. Son sólo otras emociones las que pueden modular las emociones. Por ejemplo, si el paciente se siente muy intenso avergonzado, incitar más curiosidad acerca de por qué sienten que esto podría suavizar la vergüenza. Creo que otras emociones tienen el poder de modular los sentimientos.

Sandra Toribio y Mario Nervi: En el segundo capítulo del libro Comprendiendo y tratando a pacientes en Psicoanálisis Clínico. Lecciones desde la literatura (Buechler; 2014), cuando aborda el procesamiento paranoide, en determinado momento plantea la influencia del género en su trabajo. Cito; "Me pregunto cómo afecta mi género al significado que tiene para mí ser acusada de ser demasiado optimista. A veces me siento doblemente condenada. Sé que expresa. Este material es para uso científico y profesional exclusivamente y puede contener información clínica sensible. Los editores no se responsabilizan de los contenidos de los autores. Dirigir las consultas sobre derechos y autorizaciones a ceir@psicoterapiarelacional.es 
se me verá no solo como una optimista tonta, sino también como una mujer superficial, de cabeza hueca y cerebro disperso. Creo que, en un clima paranoide, la esperanza siempre se ridiculiza. Pero mi género puede jugar algún rol en la manera en la cual mi paciente y yo experimentamos mi actitud" (pág; 38) ¿Querría hacer alguna puntualización respecto a la influencia del género y su experiencia clínica?

Sandra Buechler: En general, no creo que haya muchas veces que me discriminaron otros analistas, porque sea una mujer. Pero creo que tuvo un impacto con algunos pacientes. Por un lado, con algunos pacientes, pude decir cosas que tal vez no hubieran tolerado de un clínico masculino. Creo que era menos una amenaza para algunos. Pero, por otro lado, para otros, podría ser fácilmente descartado como "sólo" una mujer emocional, de cerebro disperso, optimista, poco realista. Por supuesto que era más fácil despertar transferencia materna, porque soy una mujer.

Sandra Toribio y Mario Nervi: A lo largo del libro Vd. incide en la pregunta que hace a sus alumnos tras la lectura de cada capítulo; ¿Quién necesita ser el clínico para ayudar a alguien con este estilo de afrontamiento (Paranoide, esquizoide, obsesivo, histérico, etc.)?, y recalca, Quien. ¿Cómo es posible preservar cierta sensación de continuidad de uno mismo, respecto a su propia personalidad ejerciendo una profesión que exige tanta flexibilidad a la hora de "ser para otros"?

Sandra Buechler: Creo que somos objetos transicionales para nuestros pacientes. Al igual que el osito de peluche, tenemos nuestros propios contornos, hay una realidad continua que no cambia, a lo que somos. Por ejemplo, siempre aprecio la verdad, con todos los pacientes. Pero al igual que el osito de peluche, cada paciente crea la Sandra Buechler que experimentan, hasta cierto punto. Tienen un impacto en lo que llamo mi textura, ya sea que los miro o se alejan, el tono de mi voz, cuánto hablo, y así sucesivamente. Siempre soy fundamentalmente yo misma en mis valores. Pero la sensación de mí es diferente con diferentes personas. Co-creamos el ambiente y la sensación de estar juntos en la habitación. Además, algunos pacientes podrían reclutar diferentes estilos de afrontamiento que tengo en mi repertorio. Este es el tema de parte de mi libro, Still Practicing.

Alejandro Ávila - Uno de los temas que vd. ha tratado últimamente refiere al envejecimiento, y a su propia decisión de dejar la práctica clínica a los 73 años. ¿Nos puede hablar de cómo ha llegado a tomar esa decisión, y de los sentimientos que suponemos ambivalentes que ha tenido que gestionar desde ese momento, aún cercano?. 
Sandra Buechler: Para esto me referiré al trabajo que os he dado sobre "Amor y odio en la terminación de una práctica"3. Cuenta algunas de mis experiencias hasta ahora. Generalmente, los sentimientos más difíciles para mí han sido los desafíos a mi identidad. ¿Quién soy ahora? ¿Cómo puedo construir el día, cuando no puedo confiar en mis viejos rituales, programación, etc. Creo que en la jubilación puede ser difícil encontrar un propósito verdadero. No me arrepiento de la decisión, ya que creo que fue correcto, pero extraño mucho las personas con las que trabajé y de la vida laboral que tuve. Fue una buena vida, $y$ me encantó el trabajo, y me pareció extremadamente significativo.

Sandra Toribio y Mario Nervi; A lo largo del libro Comprendiendo y tratando a pacientes en Psicoanálisis Clínico. Lecciones desde la literatura (Buechler; 2020) y de las exquisitas obras de la literatura que ha seleccionado para ilustrar los capítulos y las distintas formas de afrontar los dilemas de la vida, da la sensación que el desarrollo de la gente en el proceso de convertirse en persona es una sucesión de pérdidas y desilusión; ¿Tiene esa impresión? ¿Cómo hace para mantener su sonrisa?

Sandra Buechler: Sí, es una serie de pérdidas, en cierto modo. Pero no son sólo pérdidas. Debe haber un equilibrio. Esto lo tomé de Anna Freud, que un niño necesita tener suficientes ganancias positivas para seguir adelante para sentir que es bueno crecer. Que vale la pena, renunciar a los placeres de ser un "bebé" para convertirse en una persona más grande. Creo que esto es verdad toda nuestra vida. Que tenemos que sentir que vale la pena trabajar en la siguiente etapa. Es un gran desafío. Y no siempre estoy sonriendo. Tal vez su visión de mí está moldeada por la situación interpersonal jestoy sonriendo porque estoy aquí!

Silvia Jiménez: Muchas gracias, Sandra por su presencia, siempre iluminadora, y sus generosas respuestas a nuestras preguntas. ¡Esperamos verla de nuevo en España pronto!

Realizada en Ágora Relacional, Madrid, lunes 3 de Febrero de 2020.

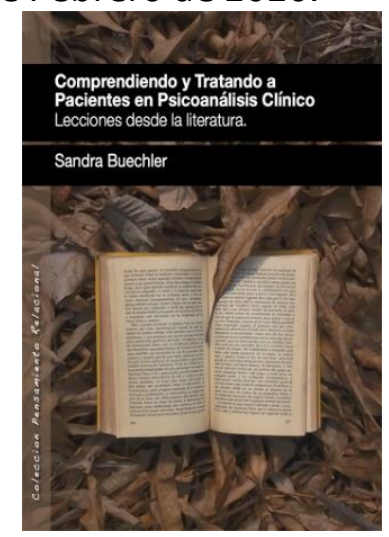

\footnotetext{
3 Este trabajo que fue presentado en la Conferencia Internacional de la IFPS en Lisboa, Febrero 2020, será publicado en castellano en el próximo número de CeIR (Octubre 2020).
} 